\title{
Editorial
}

\section{Immunisations for children and adolescents involved in competitive sports}

Sri Lanka Journal of Child Health, 2019; 48(4): 285-286

DOI: http://dx.doi.org/10.4038/sljch.v48i4.8818

(Key words: Immunisation, children, adolescents, competitive sports)

In the present-day scenario of many countries, there are significant numbers of children and adolescents involved in competitive sports. They may be called upon to compete nationally or internationally. Such requirements may involve extensive travel within the country as well as even across many a continent. Sometimes, as individuals, and at other times, as part of a team, travelling considerable distances may be a part and parcel of the life of a young sportsperson.

In that perspective, there is the possibility of these young athletes being exposed to various infectious diseases, some of which could be prevented by successful immunisation. Contracting such an infectious disease may cause many problems, including the inability to take part in the competition that they may have already arranged to take part in. Additionally, if they contract such a disease in some area of the country away from their residence, or even in another country, there may be several problems that they may have to face. Athletes are regularly exposed to crowded places, such as locker rooms, sport meetings and crowded sporting arenas, with a great number of persons around them, and in particular, for some sports, in which close contact with other sportspersons is common, all of which lead to an increased risk of airborne infection ${ }^{1}$.

Intense exercise, either acute or chronic, alters the functions and quantity of both innate and acquired immune system's circulating cells (neutrophils, monocytes and natural killer cells). The reason for the observed decrease in the acquired immune response during intense physical exercise seems to be related to an increased release and circulation of stress hormones (cortisol and catecholamines) and to the cytokines-mediated alteration of the balance of pro-inflammatory and anti-inflammatory activity $^{2}$. This alteration of the immune response following intense training and competitions is called the 'open window' and it is responsible for a higher risk of infection in athletes when exposed to microorganisms ${ }^{2}$.

However, the standard Public Health Vaccination Guidelines cannot be easily, directly or totally transferred to elite young athletes ${ }^{1}$. An enhanced benefit from preventing even mild diseases is obvious from the sports' point of view but some interference from otherwise minor side-effects, too, has to be taken into account. A risk-benefit assessment has to be undertaken on an individual basis. Such risk-benefit analyses of vaccination in elite athletes differ significantly from that of the general population, providing the rationale for specific vaccination guidelines ${ }^{3}$. Quite plainly, when living in or traveling to endemic areas, the athletes should be immune against several diseases such as tick-borne encephalitis, yellow fever, Japanese encephalitis, poliomyelitis, typhoid fever, and meningococcal disease ${ }^{1}$. Only for very few diseases would a determination of antibody titres be deemed reasonable or necessary, to avoid unnecessary vaccinations or to determine the efficacy of an individual's vaccination. This is particularly relevant for measles, mumps, rubella, varicella, hepatitis B and hepatitis A. All necessary immunisations need to be scheduled in a way that possible side-effects are least likely to interfere with competition or training. At the same time, immunological concerns must be considered when the vaccination schedule is planned, because vaccine administration during the 'open window' phase could be associated with a suboptimal immune response and reduced vaccine efficacy ${ }^{2}$. Normally, vaccinations are quite well tolerated by young athletes and the resulting antibody titres are no different to those of the general population ${ }^{3}$.

In the case of children and adolescents involved in sports, it is imperative to ensure that all the vaccines advocated by the National Expanded Programme of Immunisation (EPI) have been administered at proper times to these athletes. If deficiencies in their administration are detected, they should be corrected as soon as possible. In addition, there are some vaccines that are not included in the EPI, which may need to be given to these athletes. There are several reasons as to why this is necessary and listed below are some of these reasons which make it necessary to provide these vaccines for the young athletes.

- The possibility of a vaccine-preventable disease interfering with the current competition that the sportsperson is engaged in.

- The potential likelihood of a vaccinepreventable disease affecting the 
competitor away from home and even in another country.

- The potential for some of these diseases to cause long-term problems.

- Vaccine-preventable diseases interfering with the training schedules of the athlete or with participation in future competitions.

Risk of infection is higher in athletes due to worldwide traveling and close contact with teammates or opponents. Moreover, the potential consequences of infection are perhaps more significant, since even mild infections might be relevant as far as optimal individual performance is concerned ${ }^{2}$.

In most situations, the vaccines routinely recommended for all young athletes are:-

- Tetanus and Diphtheria

- Pertussis

- Influenza

- Hepatitis A

- Hepatitis B

- Measles, Mumps, Rubella and

- Varicella

Some of these are in the National Immunisation Programmes with children and adolescent sportspersons being already covered by the programme.

There are certain diseases for which vaccines are recommended under special circumstances and for epidemiological reasons. This is particularly so for athletes who have to travel to certain countries in which these diseases are a real problem. The recognised maladies that fall into this group are:-

- Tick-Borne Encephalitis

- Yellow Fever

- Japanese Encephalitis

- Poliomyelitis

- Typhoid and

- Meningococcal disease
There are other vaccines that are recommended when athletes have another co-morbid state or a significant underlying disorder or for athletes with different abilities. The vaccines that are recommended for this purpose are:-

- Pneumococcal vaccine and

- Haemophilus influenzae Type b (Hib) vaccine

If additional information is needed, contacting the specialised authorities on immunisation for expert advice, by Team Physicians and other medical personnel involved in the care of young sportspersons, is advocated.

\section{References}

1. Collins CJ, O'Connell B. Infectious disease outbreaks in competitive sports, 2005-2010. Journal of Athletic Training 2012; 47(5):516-8.

https://doi.org/10.4085/1062-6050-47.5.02

PMid: 23068588 PMCid: PMC3465031

2. Tafuri S, Sinesi D, Gallone MS. Vaccinations among athletes: evidence and recommendations. Expert Review of Vaccines 2017; 16(9):867-9.

https://doi.org/10.1080/14760584.2017.13

58092

PMid: 28724336

3. Gartner BC, Meyer T. Vaccination in Elite Athletes. Sports Medicine 2014; 44(10): 1361-76 https://doi.org/10.1007/s40279-014-02173

PMid: 24986118 PMCid: PMC4171584 B J C Perera orcid.org/0000-0001-7789-8793
Joint Editor

The author declares that there are no conflicts of interest

Open Access Article published under the Creative Commons Attribution CC-BY (c) (P) 